\title{
Assessing the Validity of the Cincinnati Prehospital Stroke Scale and the Medic Prehospital Assessment for Code Stroke in an Urban Emergency Medical Services Agency
}

\author{
Jonathan R. Studnek, PhD, NREMT-P, Andrew Asimos, MD, Jodi Dodds, MD, \\ Doug Swanson, MD
}

ABSTRACT

Objectives. The primary objective of this study was to assess the effectiveness of two prehospital stroke screens in correctly classifying patients suspected of having a stroke. Secondarily, differences in the sensitivity and specificity of the two screening tools were assessed. Methods. We performed a retrospective assessment of the Cincinnati Prehospital Stroke Scale (CPSS) and the Medic Prehospital Assessment for Code Stroke (Med PACS) between March 1, 2011, and September 30, 2011, in a single emergency medical services (EMS) agency with seven local hospitals all classified as stroke-capable. We obtained data for this analysis from the EMS electronic patient care reports (ePCRs) and the Get With The Guidelines ${ }^{-}$Stroke (GWTG-S) registries maintained by the two local health care systems by matching on patient identifiers. The Med PACS was developed specifically for the EMS agency under study by a local team of neurologists, emergency physicians, and paramedics. All of the physical assessment elements of the CPSS were included within the Med PACS. Two additional physical assessment items, gaze and leg motor function, were included in the Med PACS. We classified patients as CPSS-positive or -negative and Med PACS-positive or -negative if any one of the physical assessment findings was present. We determined the presence of a hospital discharge diagnosis of stroke from GWTG-S. We calculated sensitivity and specificity with resultant $95 \%$ confidence intervals. Results. We enrolled 416 patients in this study, of whom $186(44.7 \%)$ were diagnosed with a stroke. The Med PACS scale demonstrated a sensitivity of $0.742(95 \%$ confidence interval [CI] 0.672-0.802), while the sensitivity for the CPSS was 0.790 (95\% CI 0.723-0.845). The sensitivity of the CPSS was significantly higher than that of the Med PACS, with a difference of 0.048 (95\% CI 0.009-0.088; $\mathrm{p}=0.011)$. The specificities of these two scales were low, Med PACS 0.326 (95\% CI 0.267-0.391) vs. CPSS 0.239 (95\% CI 0.187-0.300),

Received December 6, 2012, from Mecklenburg EMS Agency (JRS), Charlotte, North Carolina; the Department of Emergency Medicine, Carolinas Medical Center (AA, DS), Charlotte, North Carolina; and the Department of Neurology, Presbyterian Healthcare (JD), Charlotte, North Carolina. Revision received January 14, 2013; accepted for publication January 22, 2013.

The authors report no conflicts of interest.

Address correspondence and reprint requests to: Jonathan $\mathrm{R}$. Studnek, PhD, NREMT-P, Mecklenburg EMS Agency, 4525 Statesville Road, Charlotte, NC 28269. e-mail: jonst@medic911.com doi: 10.3109/10903127.2013.773113 and the specificity of the Med PACS was significantly higher compared with the CPSS, with a difference in specificity of 0.086 (95\% CI 0.042-0.131), $\mathrm{p}<0.001$. Conclusion. The two stroke scales under study demonstrated low sensitivity and specificity, with each scale performing marginally better in one of the two metrics assessed. Key words: emergency medical services; acute stroke; prehospital stroke screens; stroke scales; screening tools; CPSS; Med PACS

PREHOSPITAL EMERGENCY CARE 2013;17:348-353

\section{INTRODUCTION}

Stroke is the fourth leading cause of mortality (40.6 per 100,000 per year) in the United States. ${ }^{1}$ Approximately 600,000 strokes are first-time events, contributing to stroke's being the leading cause of long-term disability in the United States. ${ }^{2}$ Emergency medical services (EMS) providers are a key component of the health care system and often serve as the first medical contact for stroke patients. Among the important tasks for EMS providers to perform are the recognition and identification of acute stroke patients rapidly and accurately in the field. Accurate identification allows EMS responders to initiate appropriate treatment in the field, determine the most appropriate destination facility, provide advanced notification to that facility, and expeditiously transport the patient. Prehospital advanced notification has been associated with improved stroke time targets and rates of thrombolysis. ${ }^{3-5} \mathrm{Un}$ fortunately, prehospital providers' ability to accurately identify patients presenting with stroke signs versus stroke mimics is unclear. For example, one study demonstrated that almost a fourth of the patients identified as sustaining a stroke by prehospital providers were discharged for other medical conditions. ${ }^{6}$

Prehospital stroke screens have been vigorously promoted and widely adopted to assist prehospital providers in identifying patients with acute cerebral ischemia and intracranial hemorrhage. ${ }^{7}$ The most common screens include the Cincinnati Prehospital Stroke Scale (CPSS), adapted from the hospital-based National Institutes of Health Stroke Scale for the identification of stroke in the prehospital setting, and the Los Angeles Prehospital Stroke Screen (LAPSS), developed by a prehospital and stroke expert panel. ${ }^{8,9}$ Unfortunately, the generalizability and overall performance of the LAPSS and CPSS are unclear, ${ }^{10-17}$ with 
sensitivities ranging from $44 \%$ to $91 \%$ and specificities from $33 \%$ to $97 \%$, dependent on the population under study. With the goal of providing paramedics with a tool that was both sensitive and specific in the identification of stroke patients, a local stroke screening tool was developed in Mecklenburg County, North Carolina. The Medic Prehospital Assessment for Code Stroke (Med PACS) was developed in conjunction with emergency medicine and neurology specialists. The Med PACS screening tool was created by combining the perceived strengths of the CPSS and the LAPSS.

The primary objective of this study was to assess the effectiveness in the prehospital setting of the CPSS and the Med PACS in correctly classify patients suspected of having a stroke. A secondary analysis was performed to compare the differences in the sensitivity and specificity of the two screening tools in patients experiencing signs and symptoms of acute stroke.

\section{MethodS}

We conducted a retrospective assessment of the CPSS and the Med PACS between March 1, 2011, and September 30, 2011. We included data for this analysis from the EMS electronic patient care reports (ePCRs) and the Get With The Guidelines-Stroke (GWTG-S) registries maintained by the two local health care systems. ${ }^{18}$ This study was approved by the Carolinas Medical Center institutional review board.

\section{Study Setting}

The EMS agency under study served a population of approximately 867,000 individuals with a yearly call volume averaging around 90,000, resulting in approximately 70,000 patient transports per year. Patients were transported to any of seven area hospitals based on patient preference or, in the absence of preference, the closest facility. Four hospitals were managed by one health care system and three by another. Each hospital system managed one tertiary referral hospital, inclusive of a level I trauma center. All hospitals were classified as capable of administering intravenous tissue plasminogen activator ( $\mathrm{t}-\mathrm{PA}$ ) to ischemic stroke patients during the study period and there was no stroke bypass protocol for EMS in place. All ambulances were staffed with at least one paramedic and one basic emergency medical technician (EMT). First responders within the city and county were trained at the basic EMT level and had access to an automated external defibrillator (AED). Prehospital triage, treatment, and transport protocols were uniform throughout.

In February 2011, prior to initiation of the study protocol, all paramedics received a two-hour continuing education lecture regarding neurologic emergencies. The objectives of this study were not shared with the paramedics at that time, nor were the paramedics pro- vided any additional stroke-related education or updates regarding the study during data collection.

\section{Patient Population}

We included patients in this study if they received a prehospital Med PACS screen and were transported to one of the seven local hospitals. The current EMS agency protocols stipulated that a Med PACS screen be performed on all patients who had signs or symptoms of acute stroke or transient ischemic attack (TIA). It was possible that some patients transported would not have a documented Med PACS screen, but ultimately ended up with a hospital diagnosis of stroke. We excluded these patients from the primary analysis, as an assessment of the performance of the screening tool, and not patient assessment skills, was the primary objective. We also excluded other patients, including minors (aged $<18$ years), if they were transported to any medical facility other than those in the inclusion criteria, or if they were secondary transports from a regional facility.

\section{Screening Tools}

The CPSS required providers to assess facial droop, arm drift, and speech clarity to identify stroke. The CPSS requires little training because of its simplicity and has been shown to increase recognition of stroke. ${ }^{19}$ Locally the Med PACS screening tool had been in use since April 2006. The Med PACS was developed specifically for the EMS agency under study. The screening tool was designed by a team of neurologists, emergency physicians, and paramedics from the community and approved for use by the local medical control board. The Med PACS combined elements of the CPSS and the LAPSS, along with some additional neurologic examination elements. As Figure 1 demonstrates, all of the physical assessment elements of the CPSS were included within the Med PACS, while the Med PACS items related to patient history were utilized from the LAPSS. Two other physical assessment items, gaze preference and leg motor function, were included by the local prehospital stroke subcommittee based on expert opinion. A patient was said to have met Med PACS criteria for a presumptive diagnosis of a stroke if any of the five physical assessment items were abnormal.

\section{Data Acquisition}

We obtained data from the prehospital ePCR database and the GWTG-S registries from both health care systems. For all patients meeting the inclusion criteria, we collected the Med PACS score, date of transport, date of birth, and gender. We cross-referenced these data with the GWTG-S registry from each facility to 


\begin{tabular}{|l|c|c|c|}
\hline & LAPSS & CPSS & $\begin{array}{c}\text { Med } \\
\text { PACS }\end{array}$ \\
\hline Eligibility Criteria & $\mathrm{X}$ & & \\
\hline Age over 45 years & $\mathrm{X}$ & & $\mathrm{X}$ \\
Prior history of seizure & $\mathrm{X}$ & & $\mathrm{X}$ \\
Onset $\leq 25$ hours & $\mathrm{X}$ & & \\
Ambulatory prior to event & & & \\
Blood glucose level between & $\mathrm{X}$ & & $\mathrm{X}$ \\
60 and 400 mg/dL & & & \\
\hline & & $\mathrm{X}$ & $\mathrm{X}$ \\
Examination & $\mathrm{X}$ & $\mathrm{X}$ & \\
\hline Facial smile/grimace & $\mathrm{X}$ & & $\mathrm{X}$ \\
Grip & $\mathrm{X}$ & $\mathrm{X}$ & $\mathrm{X}$ \\
Arm weakness/drift & & $\mathrm{X}$ & $\mathrm{X}$ \\
Speech & & & $\mathrm{X}$ \\
Gaze preference & & & \\
Leg weakness/drift &
\end{tabular}

FIGURE 1. Comparison of examination findings for three stroke screens. CPSS $=$ Cincinnati Prehospital Stroke Scale; LAPSS $=$ Los Angeles Prehospital Stroke Screen; Med PACS = Medic Prehospital Assessment for Code Stroke.

determine whether patients had a hospital discharge diagnosis of stroke. Data were cross-referenced using date of birth, gender, race, and time of service. GWTG-S is a hospital-based registry sponsored by the American Heart Association utilized extensively for continuous quality improvement monitoring. ${ }^{20}$ Any patient transported by EMS to one of the seven local hospitals with a discharge diagnosis of stroke or TIA was captured in one of the two health care systems' GWTG-S registries. All hospitals within the two health care systems provided registry data to a centralized repository. This method of data transfer and data capture was successfully implemented within this health care system for a study of patients suffering from severe sepsis. ${ }^{21}$

\section{Data Analysis}

After ePCR review, we classified patients as CPSSpositive or -negative and Med PACS-positive or -negative if any one of the physical assessment findings was present. For the Med PACS screen, if an eligibility criterion was positive (e.g., history of seizure disorder), then the results of the overall examination were considered negative, regardless of the physical examination findings. We classified individuals with a positive stroke screen and a stroke or TIA diagnosis in GWTG-S as true positives; those with a positive stroke screen and not in the GWTG-S registry were considered false positives. Individuals with a negative stroke screen but a final hospital diagnosis of stroke were considered false negatives. Those with a negative stroke screen who did not appear in the GWTG-S registry were considered true negatives.
Based on these classifications, we calculated sensitivity, specificity, positive and negative predictive values, and positive and negative likelihood ratios, with resultant $95 \%$ confidence intervals (CIs). We calculated these measures separately for each screening tool. We performed McNemar's chi-square analysis to compare the performances of the stroke screen tools. We conducted all statistical analysis using Stata Version 10 (StataCorp LP, College Station, TX).

Prior research had indicated that the range of sensitivity among differing stroke scales was $78 \%$ to $90 \%$ and the range of specificities was $74 \%$ to $87 \% .{ }^{21}$ We considered this prior work for determining the sample size required for this study. We targeted statistical differences in specificity to determine the sample size for this analysis. Literature indicates that specificity is the measure that is most variable, and the area in which the most increase is needed. The highest reported specificity for the CPSS was 79\%, reported by Bray et al. ${ }^{22}$ The highest reported specificity for any stroke assessment was also reported by Bray et al., at $87 \%$, which is a $10 \%$ change in specificity. With an alpha level of 0.05 and power to detect a difference of 0.80 , the sample size needed to detect a difference in the specificity reported in the literature would be 370 patients. Literature indicates that the percent change in sensitivity between the CPSS and other stroke screens is greater than $10 \%$, thereby necessitating smaller sample sizes to detect a difference. Therefore, a sample of 370 patients should be sufficient to test the difference in the sensitivity and specificity of the CPSS compared with the Med PACS.

\section{ResUlts}

We enrolled 468 patients in this study but excluded 52 of these patients because a stroke screen was incomplete or not performed, leaving 416 (88.9\%) patients eligible for analysis. Table 1 provides the descriptive statistics for the study population. The average age of the patients in this study was 66.8 (SD = 16.7). There were $226(54.3 \%)$ patients who were female and $212(51.0 \%)$ patients who were white. The Med PACS screen classified $293(70.4 \%)$ patients as potentially having an acute stroke, while the CPSS classified $322(77.4 \%)$ patients as having an acute stroke. Overall, $186(44.7 \%)$ patients were discharged from the hospital with a diagnosis of stroke, with a majority of these patients $(81.7 \%)$ discharged with a diagnosis of ischemic stroke.

Table 2 displays an assessment of the effectiveness of the screening tools in correctly classifying patients suspected of having an acute stroke. Analysis indicates that neither scale in this study as tested performed with a great degree of accuracy for appropriately classifying patients. The Med PACS scale demonstrated a sensitivity of 0.742 (95\% CI $0.672-0.802)$, while the 
TABlE 1. Sample Demographics Overall and by Diagnosis of Stroke

\begin{tabular}{|c|c|c|c|}
\hline Variable & Sample & $\begin{array}{l}\text { Diagnosis of Stroke } \\
\quad(n=186 ; 44.7 \%)\end{array}$ & $\begin{array}{c}\text { No Diagnosis of } \\
\text { Stroke }(n=230 ; \\
55.3 \%)\end{array}$ \\
\hline \multicolumn{4}{|l|}{ Gender } \\
\hline Female & $226(54.3 \%)$ & $104(46.0 \%)$ & $122(54.0 \%)$ \\
\hline Male & $190(45.7 \%)$ & $82(43.2 \%)$ & $108(56.8 \%)$ \\
\hline \multicolumn{4}{|l|}{ Race } \\
\hline White & $212(51.0 \%)$ & $96(45.3 \%)$ & $116(54.7 \%)$ \\
\hline Other & $204(49.0 \%)$ & $90(44.1 \%)$ & $114(55.9 \%)$ \\
\hline \multicolumn{4}{|l|}{ Med PACS } \\
\hline Positive & $293(70.4 \%)$ & $138(47.1 \%)$ & $155(52.9 \%)$ \\
\hline Negative & $123(29.6 \%)$ & $48(39.0 \%)$ & $75(61.0 \%)$ \\
\hline \multicolumn{4}{|l|}{ CPSS } \\
\hline Positive & $322(77.4 \%)$ & 147 (45.7\%) & $175(54.3 \%)$ \\
\hline Negative & $94(22.6 \%)$ & 39 (41.5\%) & $55(58.5 \%)$ \\
\hline
\end{tabular}

sensitivity for the CPSS was 0.790 (95\% CI 0.723-0.845). The ability of these scales, in this population, to correctly classify patients as unlikely to be experiencing a stroke, expressed as specificity, was low: Med PACS 0.326 (95\% CI $0.267-0.391)$ vs. CPSS 0.239 (95\% CI $0.187-0.300)$.

While the reported effectiveness of these scales in this study was poor, there were significant differences between the sensitivities and specificities of the scales. Using McNemar's test, the specificity of the Med PACS was significantly higher when compared with the CPSS, with a difference in specificity of 0.086 (95\% CI 0.042-0.131), p < 0.001. Conversely, the sensitivity of the CPSS was significantly higher than that of the Med PACS, with the difference being 0.048 (95\% CI 0.009-0.088), $\mathrm{p}=0.011$.

\section{Discussion}

Prehospital stroke screens are routinely recommended as an essential component of the prehospital evaluation of suspected stroke patients. ${ }^{7}$ Optimally, such screens should be both sufficiently sensitive and specific that they correctly identify the most common stroke presentations and distinguish them from stroke mimics. This has become even more important recently as states are increasingly enacting legislation or regulations permitting or directing EMS routing to statedesignated or Joint Commission-certified stroke cen- ters based on the results of prehospital stroke screens. ${ }^{23}$ Insensitive screens could result in the transport of patients to hospitals that lack the necessary resources and the capability to treat an ischemic stroke patient with thrombolytic therapy. Alternatively, bypassing hospitals based on false-positive screens has the potential to result in costly and inconvenient hospital diversion. Furthermore, especially when hospital bypass is undertaken in more rural settings with a limited number of ambulances, inappropriate diversion based on an incorrect screen may result in decreased availability of an ambulance to transport other patients requiring acute medical treatment. Fortunately, in the urban county studied, there was no bypass protocol in effect, so this was not an issue for the EMS setting studied, but certainly does pertain to other rural settings.

Although the CPSS and the LAPSS are regularly promoted as sensitive and specific, this is called into question by published data obtained outside the Cincinnati region and Southern California, respectively. In a retrospective observational study similar to ours conducted in Southern California, Ramanujam et al. found that of 477 patients with a paramedic assessment of stroke using the CPSS, 193 had a final discharge diagnosis of stroke, resulting in a sensitivity of $44 \%$ and a positive predictive value of $40 \%$ for paramedics using the CPSS. ${ }^{14}$ In a study of the LAPSS in Germany, the LAPSS performed better, but was still deemed to be "insufficient," based on a sensitivity of $68.3 \% .{ }^{15}$ From a specificity standpoint, equally suboptimal performance has been reported. In a study of the Durham County EMS system in North Carolina, Frendl et al. reported that of patients presenting with a CPSS abnormality, less than half had a final diagnosis of stroke or TIA. ${ }^{16}$ Similarly, in Australia, Bergs et al. reported a specificity of only $33 \%$ for the CPSS. ${ }^{17}$ For reasons such as these, it was reasonable to develop the Med PACS screen to determine whether sensitivity and specificity could be improved.

In our study, the Med PACS tool was inherently more complex because of the added eligibility criteria and physical examination findings. However, our results indicated that the CPSS yielded significantly increased sensitivity when compared with the Med PACS. While adding additional physical examination findings should have increased the sensitivity of the Med PACS, it is likely that the eligibility criteria eliminated any sensitivity benefit from

TABLE 2. Stroke Screen Performance

\begin{tabular}{lcccccc}
\hline Stroke Screen & Sensitivity $(95 \%$ CI $)$ & Specificity $(95 \%$ CI $)$ & PPV $(95 \%$ CI $)$ & NPV $(95 \%$ CI $)$ & LR+ $(95 \%$ CI $)$ & LR- (95\% CI) \\
\hline Med PACS & $0.742(0.672-0.802)$ & $0.326(0.267-0.391)$ & $0.471(0.413-0.530)$ & $0.610(0.518-0.696)$ & $1.10(0.973-1.24)$ & $0.791(0.582-1.07)$ \\
CPSS & $0.790(0.723-0.845)$ & $0.239(0.187-0.300)$ & $0.456(0.401-0.513)$ & $0.585(0.479-0.686)$ & $1.04(0.936-1.15)$ & $0.877(0.611-1.26)$ \\
\hline
\end{tabular}

$\mathrm{CI}=$ confidence interval; $\mathrm{CPSS}=$ Cincinnati Prehospital Stroke Scale; $\mathrm{LR}+=$ positive likelihood ratio; $\mathrm{LR}-=$ negative likelihood ratio; Med PACS $=$ Medic Prehospital Assessment for Code Stroke; NPV = negative predictive value; $\mathrm{PPV}=$ positive predictive value. 
the physical examination findings. Whereas the Med PACS had significantly better specificity than the CPSS, more recent stroke literature has suggested that the risk of administering $\mathrm{t}-\mathrm{PA}$ to a patient with a stroke mimic is minimal, while not initiating t-PA therapy in stroke patients can have significant consequences. ${ }^{24-26}$

Results from our study indicated that in an urban system where all local hospital emergency departments can deliver t-PA, a prehospital stroke screen such as the CPSS may be more useful. A screen with higher sensitivity allows for the prehospital system to alert/activate resources while lowering the probability of missing stroke patients. In a system where resources are limited and hospital bypass protocols are in place, the opposite may be true, in that a screen with greater specificity may be needed. Future prospective studies evaluating patient outcomes in a system with high-sensitivity stroke screens are needed to determine the effectiveness of resource utilization when aligned with cost.

\section{LIMITATIONS}

This study has several limitations; the generalizability of the results to other systems is an important one. The EMS system under study was urban, with all hospitals able to deliver t-PA. As such, there was no stroke bypass hospital and those patients suspected of having a stroke could be transported to any of the hospitals within the system. There were also no protocols in place related to any type of "early activation" based on prehospital assessment of stroke. The ability to transport stroke patients to any hospital may have impacted how aggressively providers utilized stroke screening tools and may account for the lower sensitivities and specificities reported in this current analysis. Furthermore, generalizability may be limited, since this was a single-center study. However, this was one of the first studies to attempt to assess the validity of the CPSS in the southeastern region of the United States.

While prehospital providers were not informed of the study purpose during annual training, they were instructed on how to adequately document patients presenting with stroke-like symptoms. However, in the ePCR, the stroke screen was not a mandatory field. Some positive strokes were identified in the GWTG-S registry that did not have a screen documented. Since the objective of this study was to evaluate the validity of the two stroke screens and not a provider's ability to recognize stroke-like symptoms, these cases were excluded. It was also likely that some patients had a stroke screen performed that was negative and therefore not recorded in the ePCR, which may have impacted the reported specificity. While data capture may have been imperfect, it likely represents the normal operation of this system and many other similar systems.

\section{Conclusions}

The two stroke scales under study demonstrated low sensitivity and specificity, with each scale performing marginally better in one of the two metrics assessed. The CPSS demonstrated statistically significant increased sensitivity and is a less-complex screening tool than the Med PACS.

\section{References}

1. Towfighi A, Saver J. Stroke declines from third to fourth leading cause of death in the United States: historical perspective and challenges ahead. Stroke. 2011;42:2351-5.

2. Roger VL, Go AS, Lloyd-Jones DM, et al. Heart disease and stroke statistics-2011 update: a report from the American Heart Association. Circulation. 2011;123(4):e18-e209.

3. Lin CB, Peterson ED, Smith EE, et al. Emergency medical service hospital prenotification is associated with improved evaluation and treatment of acute ischemic stroke. Circ Cardiovasc Qual Outcomes. 2012;5:514-22.

4. Patel MD, Rose KM, O'Brien EC, Rosamond WD. Prehospital notification by emergency medical services reduces delays in stroke evaluation: findings from the North Carolina Stroke Care Collaborative. Stroke. 2011;42:2263-8.

5. McKinney JS, Mylavarapu K, Lane J, Roberts V, OhmanStrickland P, Merlin MA. Hospital pre-notification of stroke patients by emergency medical services improves stroke time targets. J Stroke Cardiovasc Dis. 2013;22:113-8.

6. Vanni S, Polidori G, Pepe G, et al. Use of biomarkers in triage of patients with suspected stroke. J Emerg Med. 2011;40:499-505.

7. Acker JE 3rd, Pancioli AM, Crocco TJ, et al. Implementation strategies for emergency medical services within stroke systems of care: a policy statement from the American Heart Association/American Stroke Association Expert Panel on Emergency Medical Services Systems and the Stroke Council. Stroke. 2007;38:3097-115.

8. Kothari R, Hall K, Brott T, Broderick J. Early stroke recognition: developing an out-of-hospital NIH Stroke Scale. Acad Emerg Med. 1997;4:986-90.

9. Kidwell CS, Saver JL, Schubert GB, Eckstein M, Starkman S. Design and retrospective analysis of the Los Angeles Prehospital Stroke Screen (LAPSS). Prehosp Emerg Care. 1998;2:267-73.

10. Harbison J, Hossain O, Jenkinson D, Davis J, Louw SJ, Ford GA. Diagnostic accuracy of stroke referrals from primary care, emergency room physicians, and ambulance staff using the face arm speech test. Stroke. 2003;34:71-6.

11. Nor AM, McAllister C, Louw SJ, et al. Agreement between ambulance paramedic- and physician-recorded neurological signs with Face Arm Speech Test (FAST) in acute stroke patients. Stroke. 2004;35:1355-9.

12. Kidwell CS, Starkman S, Eckstein M, Weems K, Saver JL. Identifying stroke in the field. Prospective validation of the Los Angeles Prehospital Stroke Screen (LAPSS). Stroke. 2000;31:71-6.

13. Kothari RU, Pancioli A, Liu T, Brott T, Broderick J. Cincinnati Prehospital Stroke Scale: reproducibility and validity. Ann Emerg Med. 1999;33:373-8.

14. Ramanujam P, Guluma KZ, Castillo EM, et al. Accuracy of stroke recognition by emergency medical dispatchers and paramedics-San Diego experience. Prehosp Emerg Care. 2008;12:307-13.

15. Ziegler V, Rashid A, Müller-Gorchs M, et al. Mobile computing systems in preclinical care of stroke. Anaesthesist. 2008;57:677-85.

16. Frendl DM, Strauss DG, Underhill BK, Goldstein LB. Lack of impact of paramedic training and use of the Cincinnati 
Prehospital Stroke Scale on stroke patient identification and onscene time. Stroke. 2009;40:754-6.

17. Bergs J, Sabbe M, Moons P. Prehospital stroke scales in a Belgian prehospital setting: a pilot study. Eur J Emerg Med. 2010;17:2-6.

18. Schwamm LH, Fonarow GC, Reeves MJ, et al. Get With the Guidelines-Stroke is associated with sustained improvement in care for patients hospitalized with acute stroke or transient ischemic attack. Circulation. 2009;119:107-15.

19. Hurwitz AS, Brice JH, Overby BA, Evenson KR. Directed use of the Cincinnati Prehospital Stroke Scale by laypersons. Prehosp Emerg Care. 2005;9:292-6.

20. Xian Y, Fonarow GC, Reeves MJ, et al. Data quality in the American Heart Association Get With The Guidelines-Stroke (GWTG-Stroke): results from a national data validation audit. Am Heart J. 2012;163:392-8.

21. Studnek JR, Artho MR, Garner CL Jr, Jones AE. The impact of emergency medical services on the ED care of severe sepsis. Am J Emerg Med. 2012;30:51-6.
22. Bray JE, Martin J, Cooper G, Barger B, Bernard S, Bladin C. Paramedic identification of stroke: community validation of the Melbourne Ambulance Stroke Screen. Cerebrovasc Dis. 2005;20:28-33.

23. Song S, Saver J. Growth of regional acute stroke systems of care in the United States in the first decade of the 21st century. Stroke. 2012;43:1975-8.

24. Chernyshev OY, Martin-Schild S, Albright KC, et al. Safety of tPA in stroke mimics and neuroimagingnegative cerebral ischemia. Neurology. 2010;74: 1340-5.

25. Tsivgoulis G, Alexandrov AV, Chang J, et al. Safety and outcomes of intravenous thrombolysis in stroke mimics: a 6-year, single-care center study and a pooled analysis of reported series. Stroke. 2011;42:1771-4.

26. Wardlaw JM, Murray V, Berge E, et al. Recombinant tissue plasminogen activator for acute ischaemic stroke: an updated systematic review and meta-analysis. Lancet. 2012;379: 2364-72. 
Copyright of Prehospital Emergency Care is the property of Taylor \& Francis Ltd and its content may not be copied or emailed to multiple sites or posted to a listserv without the copyright holder's express written permission. However, users may print, download, or email articles for individual use. 